\title{
Les directives de l'ASSM «Mesures de soins intensifs» mises en consultation
}

Académie Suisse des Sciences Médicales (ASSM)

Correspondance:

Académie Suisse des Sciences Médicales (ASSM)

Petersplatz 13

CH-4051 Bâle

mail[at]samw.ch
Depuis la publication des premières directives de l'ASSM «Problèmes éthiques aux soins intensifs» en 1999, les possibilités de maintenir un patient en vie grâce à des moyens techniques et des médicaments se sont développées. En même temps, les attentes de la population à l'égard des possibilités thérapeutiques jusqu'à un âge avancé ont, elles aussi, évolué. De nos jours, la médecine intensive est en proie à des tensions croissantes entre, d'un côté, les exigences des patients et de leurs proches et de l'autre celles des médecins prescripteurs; ces tensions sont également alimentées par les questionnements éthiques concernant la pertinence de certaines interventions ainsi que les limites de financement imposées par la politique, les assurances et l'administration. Parallèlement, la pénurie de personnel pèse d'autant plus lourd que la médecine intensive doit de plus en plus souvent assumer des tâches qui, en fait, n'entrent pas dans son domaine d'application. Dans ce champ de tensions, il est nécessaire de définir des bases et des recommandations claires pour une utilisation

\section{Sous-commission responsable}

Prof. Reto Stocker, médecine intensive, Zurich, Président

Prof. Michel Berner, pédiatrie/néonatologie, Genève

Dr Isabelle Binet, néphrologie, St-Gall

Dr Ulrich Bürgi, médecine d'urgence, Aarau

Prof. Johannes Fischer, éthique, Zurich

Valérie Gardaz, infirmière aux soins intensifs, Genève

Dr Daniel Grob, gériatrie aiguë, Zurich

Ursula Hager, MAE, infirmière aux soins intensifs,

Zurich

Dr Christian Kätterer, médecine de réhabilitation, Bâle

Prof. Christian Kind, pédiatrie/néonatologie, St-Gall, Président CCE

Prof. Bara Ricou, médecine intensive, Genève

lic. iur. MAE Michelle Salathé, Bâle, ASSM

PD Dr Stefan Wildi, chirurgie, Zurich
Le 29 novembre 2012, le Sénat de I'ASSM a approuvé le projet de directives pour sa mise en consultation. La procédure de consultation dure jusqu'à fin février 2013. Les personnes et institutions intéressées sont invitées à s'exprimer à ce sujet. Les directives peuvent dès à présent être consultées sur le site de l'ASSM: www.samw.ch/ fr/Ethique/Directives/en-consultation.html

efficiente des mesures de soins intensifs. Les directives de 1999 ne pouvaient pas encore tenir compte de cette évolution. C'est pourquoi, en mars 2010, la Commission Centrale d'Ethique a chargé une souscommission, dirigée par le Prof. Reto Stocker de Zurich, de la révision complète des directives «Problèmes éthiques aux soins intensifs».

Le domaine d'application des directives révisées englobe toutes les mesures de soins intensifs mises en ouvre pour le diagnostic et le traitement de situations et de maladies dans lesquelles le pronostic vital est engagé. Les mesures de soins intensifs sont le plus souvent appliquées dans une unité de soins intensifs, mais il arrive fréquemment que l'on ait aussi recours à ces mesures dans des situations d'urgence préclinique, au service des urgences ou dans d'autres services; c'est pourquoi elles font partie du domaine d'application de ces directives, même en-dehors de l'unité de soins intensifs. Le projet de directives rappelle les objectifs et l'étendue des soins intensifs et définit les notions de base importantes pour la prise de décision. Pour conclure, les conséquences pour la mise en œuvre dans les différents domaines médicaux sont exposées. Les directives insistent particulièrement sur les processus de décisions au sein de l'unité de soins intensifs (par ex. lors de l'admission, du transfert et du triage). Dans l'annexe des directives, les systèmes de scoring usuels sont décrits et des recommandations pour la mise en œuvre des directives sont formulées. 